\title{
Self-Similar Prefractal Frequency Selective Surfaces for Multiband and Dual-Polarized Applications
}

\author{
John P. Gianvittorio, Student Member, IEEE, Jordi Romeu, Member, IEEE, Sebastián Blanch, and \\ Yahya Rahmat-Samii, Fellow, IEEE
}

\begin{abstract}
Frequency-selective surfaces (FSS) that have been designed using the iterative techniques of fractals have been fabricated and measured. Fractals contain many scales of the starting geometry, each of which acts as a scaled version of the original. A multiband FSS can be designed that uses several iterations of the geometry to form a prefractal that resonates corresponding to each of the scales present in the geometry. Minkowski and Sierpinski Carpet fractals have been utilized in the design of three surfaces which exhibit two or three stopbands depending on how many iterations are used to generate the geometry of the cell. These surfaces are dual polarized due to the symmetry of the geometry. Simulation capabilities have been developed to analyze these periodic structures including periodic method of moments (MOM) and finite-difference time-domain (FDTD) techniques which show good correlation to the measured results.
\end{abstract}

Index Terms-Fractals, frequency selective surface (FSS), multifrequency antennas.

\section{InTROduction AND DeSign Methodology}

$\mathbf{F}$ RACTALS, which are a modern development of geometry that define a class of objects, can be created using an iterative methodology [1]. A fractal starts as a simple geometry. A linear transformation, usually involving copying, scaling, and translation, is applied to this structure. The transformation is then applied again to the entire resulting structure. The fractal is generated by repeating this methodology an infinite number of times while a prefractal is the resulting structure if the iterative process is truncated after a finite number of times. The manufacturable fractal objects themselves must result from a truncated generation process and therefore are referred to as prefractals to be more precise.

It is the purpose of the authors to use these prefractals, which contain many scaled versions of the original simple geometry, as a frequency-selective surface (FSS). A frequency selective surface is a planar periodic structure that has a frequency response to radiation passed through it that correlates to the spacing of

Manuscript received May 15, 2002; revised October 10, 2002. This work was supported in part by the National Science Foundation (NSF) under Grant DMI-0089095, the Spanish Commission for Science and Technology (CICYTTIC2001-2364-C03-01) and in part by the Departament d'Universitats, Recerca i Societat de la Informació (DURSI).

J. P. Gianvittorio and Y. Rahmat-Samii are with the Department of Electrical Engineering, Engineering IV 64-118, University of California, Los Angeles, Los Angeles, CA 90095-1594 USA (e-mail: johng@ee.ucla.edu; rahmat@ee.ucla.edu).

J. Romeu is with the Centre Technologies de Telecomunicacio, de Catalunya (CTTC), Universitat Politécnica de Catalunya, 08034 Barcelona, Spain.

S. Blanch is with the Department of Signal, Theory and Communications,

Universitat Politécnica de Catalunya, 08034 Barcelona, Spain.

Digital Object Identifier 10.1109/TAP.2003.818791 the elements. Radiation is either allowed to transmit through or blocked depending on the retransmitted phase of the radiation from the excited elements with the same underlying fundamental principles as array theory. A frequency selective surface has a signature that, in general, is dependent on the frequency of the incident wave, the incident angle, and the incident polarization.

Several iterations of the fractal can be used to design an FSS that has a multiband frequency response that correlates to the scales of the geometry that is present in the structure. The Sierpinski Sieve fractal, utilized previously as a multiband monopole [2], has been used to design a multiband FSS previously [3], [4], which has also been utilized as a radome [5]. However, this particular structure is sensitive to only one polarization. Various prefractal FSS configurations have also been designed that can be dual-polarized due to the symmetry in the geometry [6], [7]. The fractal property of having multiple scales of similar geometry present in the final structure has also been incorporated with dipoles [8].

In this paper, the design, simulation, fabrication and measurement of prefractal surfaces based on the Minkowski and Sierpinski Carpet fractals are discussed. The simulation methods that have been developed for complex periodic structures are used to analyze these structures and are verified with measured results. These methods include a periodic method of moments (MOM) as well as a periodic finite-difference time-domain (FDTD) technique. The measurement techniques to acquire the frequency and angular responses are also covered. A flow chart outlining what is discussed herein is shown in Fig. 1. This paper expounds the entire design process for a prefractal FSS which would contain a selectable number of resonances which are responsive to both polarizations.

\section{Simulation TeChNiQues, VerificAtion, AND MEASUREMENT TECHNIQUES}

\section{A. Simulation Methods}

To simulate these electrically large and periodic structures, two approaches have been developed at the University of California, Los Angeles (UCLA). The first method uses a MOM simulation technique that incorporates periodic boundary conditions [9]. This allows for only one element of the periodic array to be simulated. When studying intricate elements such as fractals, this saves time and allows wide frequency sweeps that for some cases would not otherwise fit into the limitations of the computing hardware. While this particular periodic MOM simulation technique allows freedom to simulate tilted surfaces and 


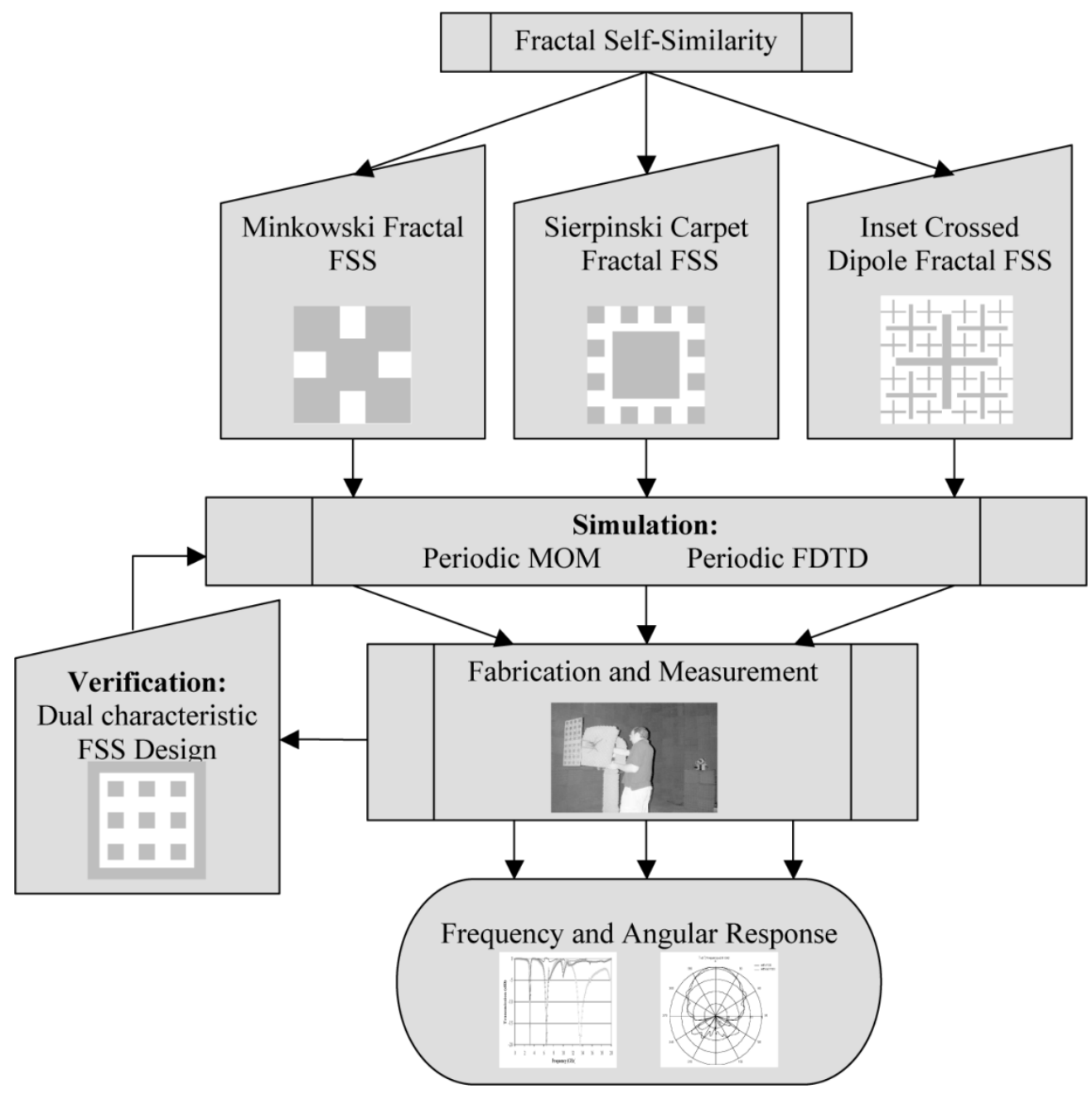

Fig. 1. Outlined in this paper is the design and analysis of three prefractal frequency selective surfaces. The structures are simulated using the periodic MOM and periodic FDTD techniques. Both methods are verified using measured data. The frequency and angular responses for the surfaces are attained.

very low incident angles, dielectrics are not incorporated. The supporting dielectric of an FSS can play a significant role in the frequency response. For dielectrics that are thicker than $1 / 10$ of a wavelength, a safe estimation of the response is to use the average of the dielectric constants on either side of the FSS, i.e. air and the supporting dielectric. The corresponding shift in frequency can then be expressed as

$$
\lambda_{\text {effective }}=\lambda_{0} \sqrt{\frac{1+\varepsilon_{r}}{2}}
$$

where $\lambda_{o}$ is the free space wavelength, $\varepsilon_{r}$ is the relative dielectric constant, and $\lambda_{\text {effective }}$ is the effective scaled wavelength [4].

Another method which can also simulate the supporting dielectric, as well, is the FDTD technique. The version of this methodology incorporates periodic boundary conditions allowing periodic arrays of complex elements to be simulated [10].

A comparison between the two simulation techniques and measured data is shown in Fig. 2. A dipole FSS is simulated using both techniques with and without a supporting dielectric. The simulations consider an infinite array of $12 \mathrm{~mm}$ by $3 \mathrm{~mm}$ perfectly electric conducting rectangular planar dipoles spaced $15 \mathrm{~mm}$ apart in both directions. The supporting dielectric is 6

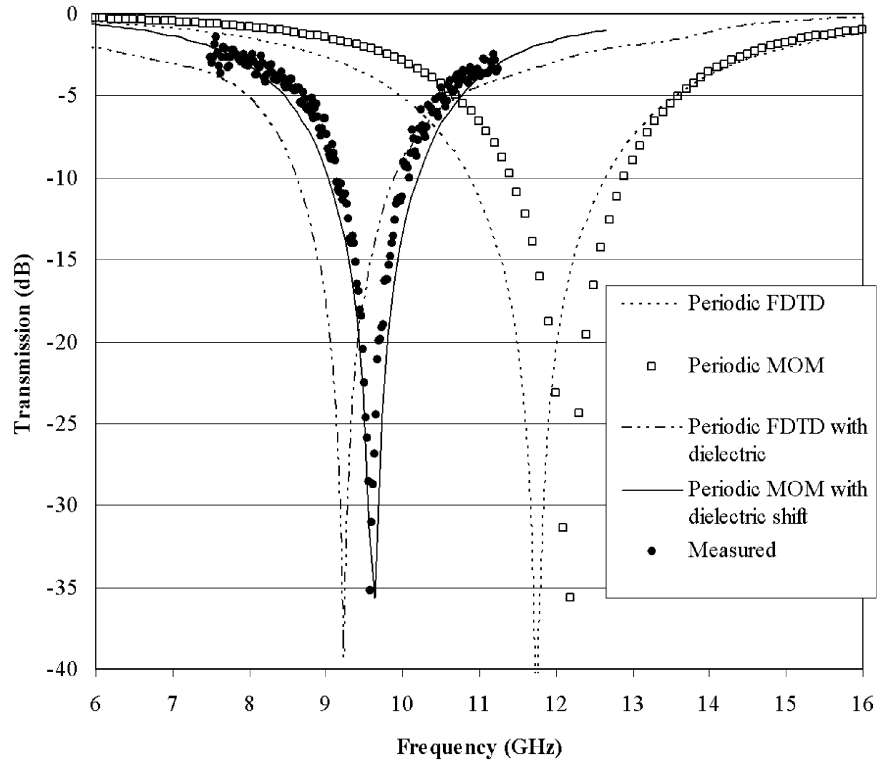

Fig. 2. Comparison between simulation of dipole FSS using periodic MOM and periodic FDTD techniques with and without the supporting dielectric and the measured results. The dielectric can be simulated using the periodic FDTD method and is incorporated into the periodic MOM simulation using an average dielectric shift. The dipole is $12 \mathrm{~mm}$ by $3 \mathrm{~mm}$ and the array spacing in both directions is $15 \mathrm{~mm}$. The measured supporting dielectric is $5.25 \mathrm{~mm}$ thick and is $6 \mathrm{~mm}$ for the FDTD simulation with a dielectric constant of 2.2. 


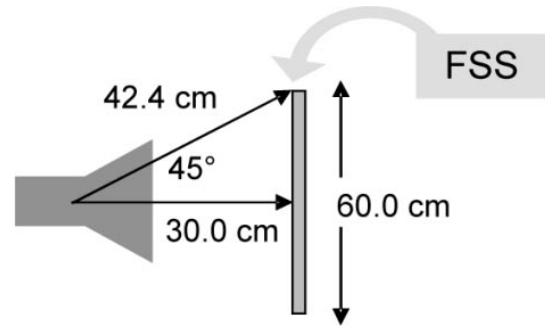

Receiving Horn in Far field

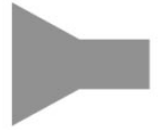

Fig. 3. Block diagram of the measurement setup used to measure frequency selective surfaces (FSS). Electromagnetic waves are transmitted through the FSS located between two horn antennas.

mm thick with a dielectric constant of 2.2. It can be seen from the plot of the computed transmission coefficients that both the periodic MOM simulation and the periodic FDTD method are in good agreement with each other and the measured data, including the simulation utilizing an average dielectric shift to incorporate the supporting dielectric. The resonant frequency calculated using the periodic MOM is shifted $4 \%$ higher than that computed using the FDTD technique for both with and without the dielectric. The measured $-10 \mathrm{~dB}$ bandwidth is $8.8 \%$ and is $11 \%$ with and without the dielectric as calculated with the periodic MOM technique and $14 \%$ and $15 \%$ with and without the dielectric, respectively, as calculated with the periodic FDTD method.

\section{B. Fabrication and Measurement Techniques}

The surfaces are fabricated using standard printed circuit technology on copper clad substrates with one side of the copper being completely removed. Enough periods of the array need to be fabricated such that the resulting structure can appear to be infinite in extent to the incident radiation. The negative effect of radiation diffracting from the edges of the screen can be mitigated by tapering the power that is directed at the edges. Typically a minimum of 16 to 36 elements are required for the measurement configurations used.

Two methods were utilized to fabricate the screens tested in this paper. The first method is to print the patterns on $1.5 \mathrm{~mm}$ thick Duroid with a dielectric constant of 2.2. A minimum of one hundred elements in a 10 by 10 array are printed onto the substrate with the copper from one side removed. The geometry is scaled such that the highest resonance would occur between 33-75 GHz, the measurable frequency range using the setup at UCLA, which consisted of a network analyzer with a millimeter wave external setup for measuring higher frequencies. The FSS is positioned on an optical bench between two horn antennas connected to the ports of the network analyzer. The lower resonances were not measurable due to strong reflections at lower frequencies from the surrounding surfaces that would obfuscate the measurement, but could be predicted with the simulation methods.

The second fabrication method utilized the facilities at the Universitat Politécnica de Catalunya (UPC). The screen is printed onto two $60 \mathrm{~cm}$ by $60 \mathrm{~cm}$ pieces of FR4, which are 1.5 $\mathrm{mm}$ thick and whose dielectric constant is 4 . At least 4 by 4 elements are printed on each screen and the two screens are taped together side by side to form a 4 by 8 array. These arrays are scaled such that all resonances occur in a frequency band of $2-14 \mathrm{GHz}$, the measurable band of the anechoic chamber.

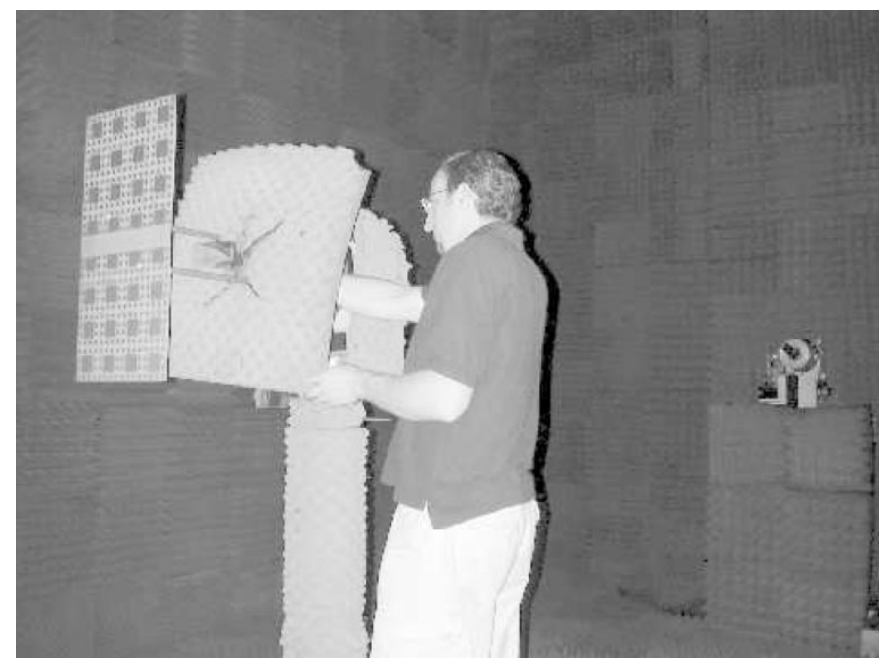

Fig. 4. Sierpinski Carpet fractal FSS supported with dielectric rods in front of a receiving horn antenna in the anechoic chamber at the Universitat Politécnica de Catalunya (UPC). This setup is used to measure the frequency and angular response of the surface.

This method also provides an adequate method for measuring the angular response of the FSS. A block diagram of the measurement setup is shown in Fig. 3. A horn antenna is used to illuminate the surface whose transmitted radiation is collected by a second horn antenna. Because the screen is only $30 \mathrm{~cm}$ from the horn antenna, the waves impinging on the surface have a spherical front and are not plane waves. The closest edge of the screen is $45^{\circ}$ from the boresight of the horn antenna. The radiation at this angle is $15 \mathrm{~dB}$ below that of the radiation at the boresight. This mitigates the diffraction from the edges of the surface that could distort the patterns. The radiation of the horn antenna drops only $5 \mathrm{~dB}$ at $\pm 30^{\circ}$. Therefore it can be concluded that the screen must be effective for incident angles up to $\pm 30^{\circ}$ in this setup. The narrower side of the array is aligned with the narrower beam of the horn antenna to mitigate the edge diffraction. The comparison between the far field radiation patterns of the horn antenna alone and with the screen in place can show the effect of the FSS. A photo of the far field chamber where these measurements were made is shown in Fig. 4. Only one polarization needs to be measured for these FSSs due to the polarization symmetry in the geometry of the FSS.

\section{Verification With Dual Characteristic Inset FSS}

An FSS is designed to have two characteristics present in the same geometry. The design consists of a screen of square holes that act as a high pass filter since the material appears to be a 


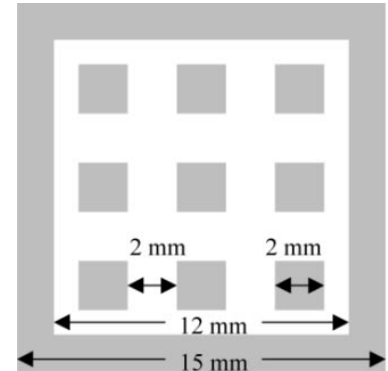

a

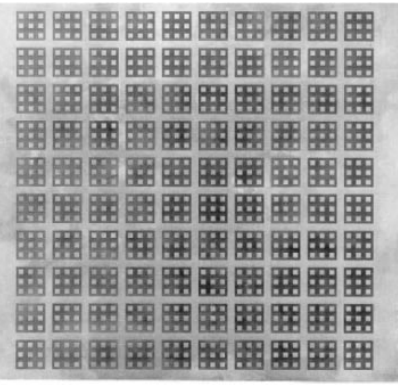

Fig. 5. (a) Periodic cell of a dual characteristic inset FSS. The grid generates a high pass response while the patches printed inside the holes generate a stopband in this passband. (b) Fabricated array printed on a Duroid substrate.

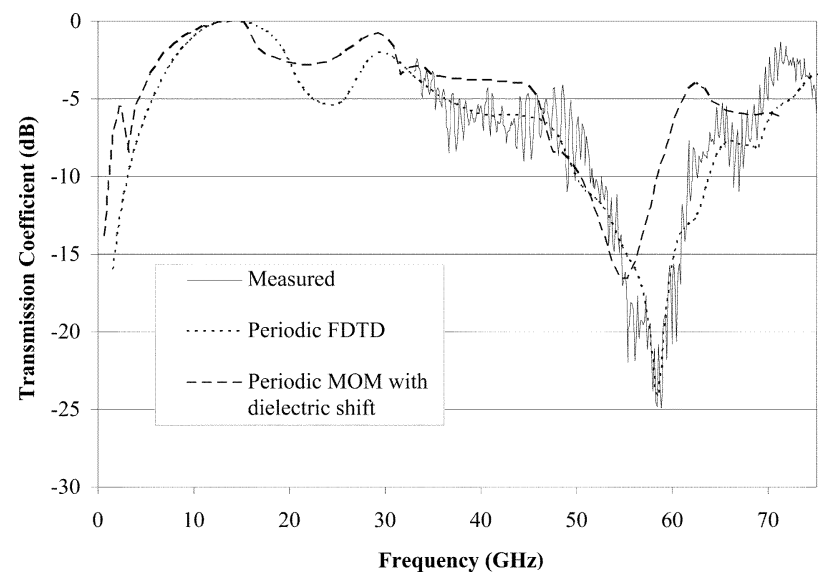

Fig. 6. Measured and computed frequency response of the dual characteristic inset FSS for normal incidence. The response is simulated using a periodic MOM technique incorporating an average dielectric shift and a periodic FDTD algorithm.

continuous PEC at wavelengths that cannot discern the holes. However, inside the holes an array of patches have been assembled that will generate a stopband in the passband of an FSS of the holes alone. The geometry is shown in Fig. 5(a). This FSS shows the feasibility of having separate geometrical features in the screen for the design of multiband structures. This design also helps to lay the groundwork and show the feasibility for prefractal FSSs.

The dual characteristic inset FSS is designed to block frequencies below $10 \mathrm{GHz}$ in addition to a stopband that is inserted near $60 \mathrm{GHz}$. The higher frequency is chosen to be within the measurable range at the facilities at UCLA. The fabricated array consists of a 10 by 10 array of cells printed onto copper clad Duroid whose dielectric constant is 2.2 with one side of copper completely removed. A photograph of the fabricated screen is shown in Fig. 5(b).

The resulting transmission coefficient for a normally incident plane wave is shown in Fig. 6. The measured data shows good correlation with the data calculated using the periodic FDTD technique. The resonant frequency as predicted with the periodic MOM technique using the average dielectric shift has resulted in an $8 \%$ downward shift, which is within acceptable tolerances for this approximation of the effect of the $1.5 \mathrm{~mm}$ supporting dielectric. Both methods accurately predict the transmission features specified in the design including rejecting low

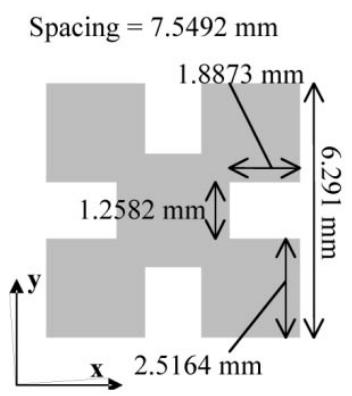

a

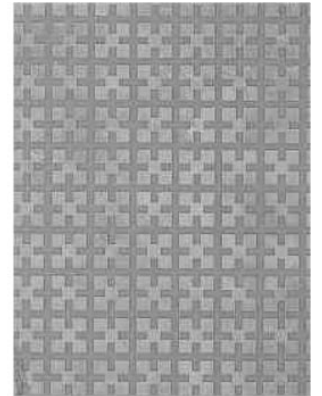

b
Fig. 7. (a) Periodic cell of a Minkowski fractal FSS. The array generates two stopbands that correspond to the overlying square without the notches and to the smaller squares formed by the notches. (b) Fabricated array that has been printed on a Duroid substrate.

frequency transmissions and rejecting another higher band of frequencies.

\section{PRefractal FSS Designs}

\section{A. Minkowski Fractal Element FSS}

1) Minkowski Fractal Element FSS Design: This design of this element for an FSS is created from a Minkowski fractal, which results in multiple resonances due to the number of iterations used to generate the prefractal element. It is generated by an iterative technique involving scaling the starting geometry and copying this four times to the corners of the structure. A square is used as the starting structure. Each iteration involves scaling and translating the previous iteration. This prefractal element requires five transformations. The resulting points of the geometry can be expressed as

$$
\begin{aligned}
m_{1}(x, y)= & \frac{2}{5}(x, y) \\
m_{2,3,4,5}(x, y)= & \frac{2}{5}\left(x \pm \frac{3}{10}, y \pm \frac{3}{10}\right) \\
M_{S+1}= & m_{1}\left(M_{S}\right) \cup m_{2}\left(M_{S}\right) \cup m_{3}\left(M_{S}\right) \\
& \cup m_{4}\left(M_{S}\right) \cup m_{5}\left(M_{S}\right)
\end{aligned}
$$

where $M_{s+1}$, the resulting set of points for the $s+1$ iteration is composed of the union of the sets of points resulting from the five transformations, $m_{1}$ through $m_{5}$. This process can continue for as many bands are desired, limited by manufacturing tolerances and measurability.

Each iteration corresponds to a stopband in the frequency response relating to the resonant squares. It should also be noted that the spacing between the elements is also scaled with each iteration. However, only the starting geometry and the first iteration have a uniform periodicity between unit cells. After the second iteration, the spacing between the resonant squares present inside one unit cell and between periods begins to vary since the spacing between the unit cells is dictated by the starting geometry.

2) Minkowski Fractal Element FSS Performance: One iteration of the fractal is studied to show the concept. This prefractal element is shown in Fig. 7(a). The structure exhibits two distinct stopbands in its transmission response. The first stopband corresponds to the larger overlying square ignoring the notches. 


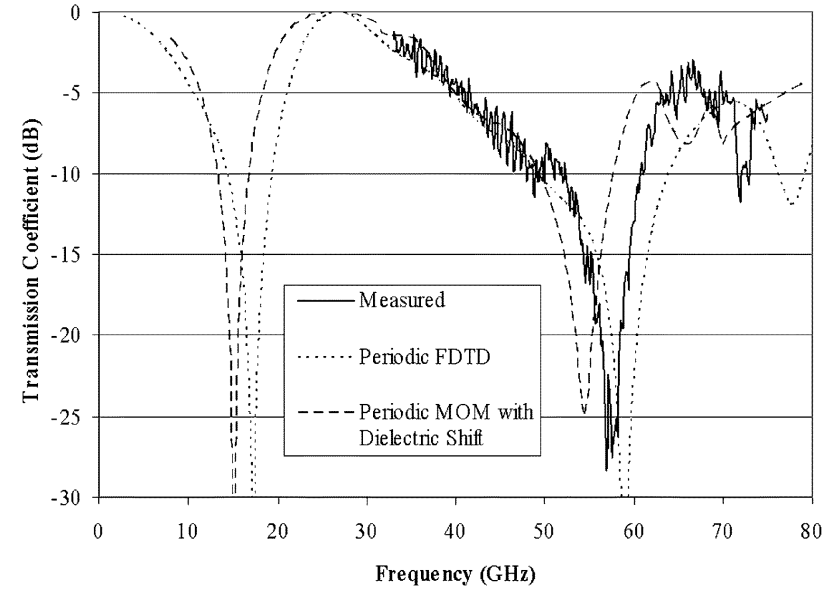

Fig. 8. Measured and simulated frequency response for the Minkowski fractal FSS for normal incidence. The measured response is compared with the simulated responses that have been attained using the periodic FDTD technique and the periodic MOM technique which incorporates a shift in frequency corresponding to an average dielectric constant.

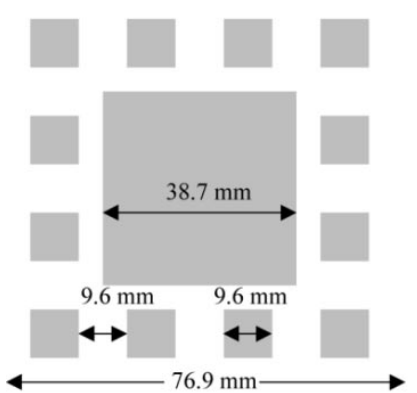

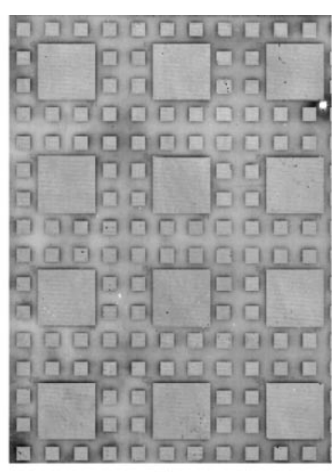

Fig. 9. (a) Periodic cell of a Sierpinski Carpet fractal FSS. The grid generates two stopbands that correspond to the two scales of resonant squares. (b) Fabricated array on fiberglass.

The second stopband corresponds with the smaller squares that are embedded inside the geometry due to the notches.

This FSS is scaled to have the second stopband near $60 \mathrm{GHz}$. A 20 by 26 array is printed on copper clad Duroid and is shown in Fig. 7(b). The computed transmission coefficients are compared with the measured data for the higher stopband in Fig. 8. The two stopbands near 15 and $55 \mathrm{GHz}$ are spaced by a factor of 3.67 while the original design called for a spacing of 2.5 . This discrepancy can be attributed to the self-similarity in the geometry being only approximate rather than exact. The smaller squares are only similar to the larger squares in their overlying geometry; however, their electrical connections are different.

\section{B. Sierpinski Carpet Fractal FSS}

1) Sierpinski Carpet Fractal FSS Design: Another fractal that can be used to design a multiband FSS where the scales in the geometry are more similar is the Sierpinski Carpet. The prefractal contains separate grids of resonant square patches that have no electrical connection between each other. Each scale of the geometry corresponds to a stopband. Also, the spacing for all the scales of the geometry remains constant with increasing iterations. This number of bands is only limited by manufacturing tolerances that dictate how small each square can be printed.

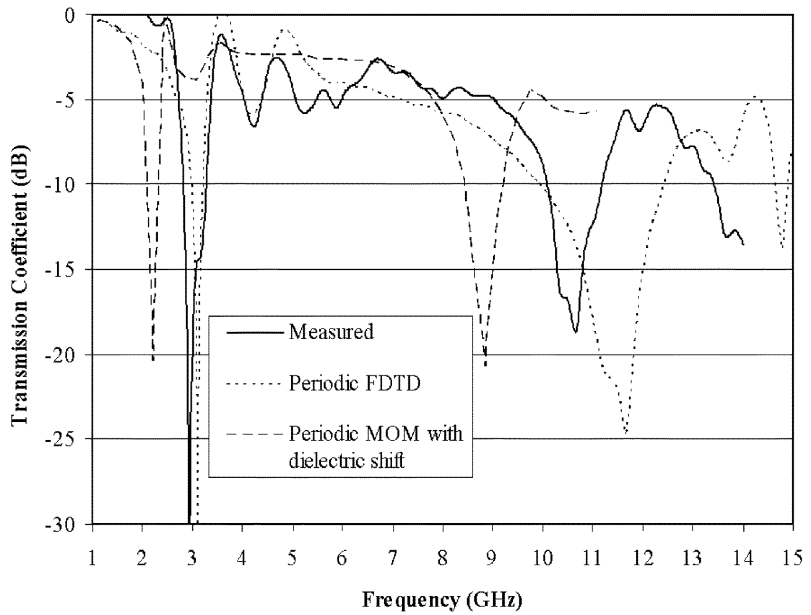

(a)

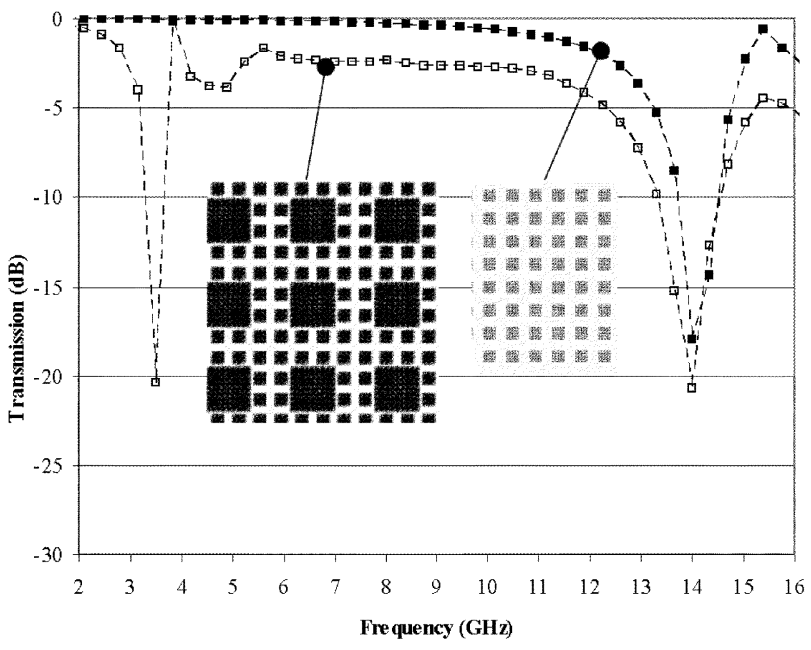

(b)

Fig. 10. (a) Measured and simulated frequency response of the Sierpinski Carpet fractal FSS. (b) Comparison of the simulated response from the periodic MOM technique of an array of only the smaller squares corresponding to the second stopband and the entire FSS.

This fractal contains a scaled version of the starting geometry and twelve scaled copies around it that are each a quarter size of the former. This can be expressed as

$$
\begin{aligned}
s_{1,2,3,4}(x, y)= & \frac{1}{4}\left(x \pm \frac{3}{8}, y \pm \frac{3}{8}\right) \\
s_{5,6,7,8}(x, y)= & \frac{1}{4}\left(x \pm \frac{1}{8}, y \pm \frac{3}{8}\right) \\
s_{9,10,11,12}(x, y)= & \frac{1}{4}\left(x \pm \frac{3}{8}, y \pm \frac{1}{8}\right) \\
C_{S+1}= & C_{S} \cup s_{1}\left(C_{S}\right) \cup s_{2}\left(C_{S}\right) \cup s_{3}\left(C_{S}\right) \\
& \cup s_{4}\left(C_{S}\right) \cup s_{5}\left(C_{S}\right) \cup s_{6}\left(C_{S}\right) \\
& \cup s_{7}\left(C_{S}\right) \cup s_{8}\left(C_{S}\right) \cup s_{9}\left(C_{S}\right) \\
& \cup s_{10}\left(C_{S}\right) \cup s_{11}\left(C_{S}\right) \cup s_{12}\left(C_{S}\right)
\end{aligned}
$$

where $C_{s+1}$, the resulting set of points for the $s+1$ iteration is composed of the union of the sets of points resulting from the twelve transformations, $s_{1}$ through $s_{12}$.

2) Sierpinski Carpet Fractal FSS Performance: One iteration of the Sierpinski Carpet fractal has been fabricated to show the feasibility of using this structure as a multiband FSS. The 

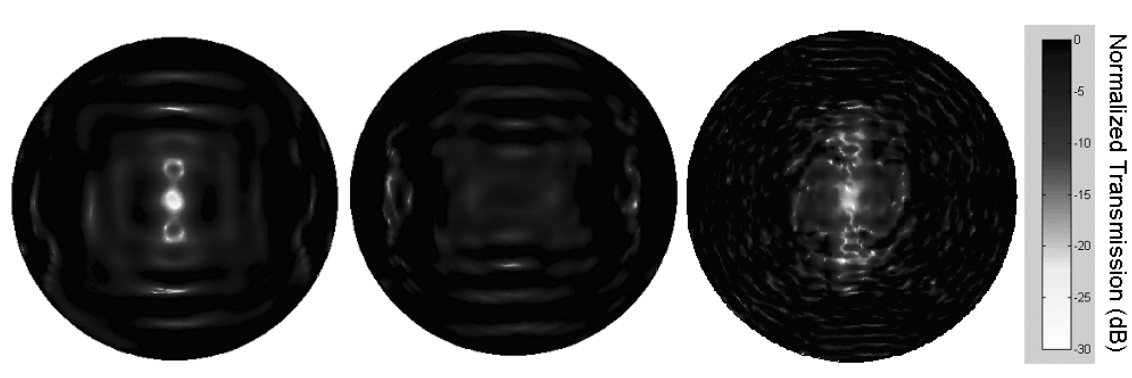

(a)
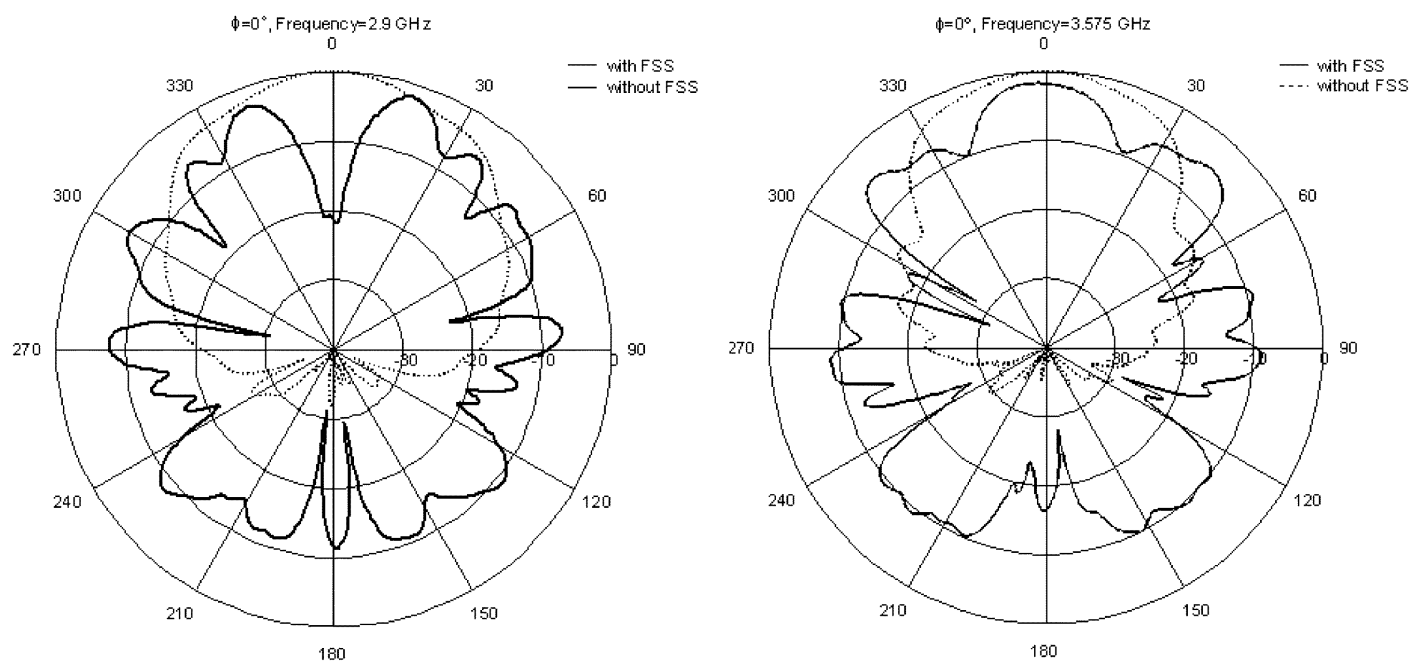

(b)

Fig. 11. Measured far field patterns of the Sierpinski Carpet fractal FSS. The measured patterns at the three bands of interest (a)starting from the left: the first stopband, the passband between the two stopbands, and the second stopband. (b) Cuts of the first stopband and first passband.

element of one iteration of the fractal is shown in Fig. 9(a) and the fabricated screen is shown in Fig. 9(b). The fabricated screen has been printed on a piece of copper clad FR4. The screen is scaled for the two stopbands to be at 3.5 and $14 \mathrm{GHz}$, which match the measurement setup. Two panels that contain 4 by 4 prefractal elements have been fabricated and are connected next to each other when measured in the far field chamber.

The frequency responses of the transmission coefficient attained through calculation and measurement are plotted in Fig. 10(a). The two stopbands as calculated with the periodic MOM simulation are spaced four times apart from each other as predicted from the design. However, it can be seen that the measured resonant frequencies are shifted due to the supporting dielectric. In general the dielectric constant of a material is frequency dependent and can only be considered constant over a very narrow frequency band [11]. The dielectric constant of the supporting FR4 is, therefore, not steady over the frequency range that is measured and since the dielectric is very thin, $1.5 \mathrm{~mm}$, the predicted shift using the average dielectric is less valid. However, the features of the measured results are consistent with what was predicted from the simulation. Also, it can be seen that the losses in the passband between the two stopbands is much higher than would typically be useable for some applications. The simulated results shown in Fig. 10(b) attained using the periodic MOM technique show the calculated response of an array of squares and the first iteration of the fractal. It can be seen that the addition of an iteration to the

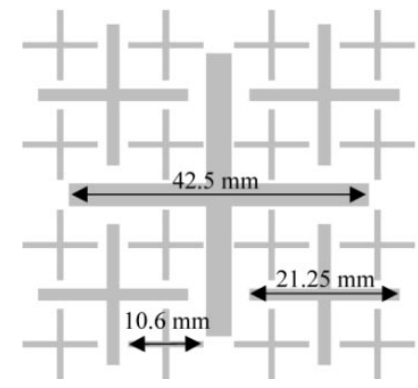

(a)

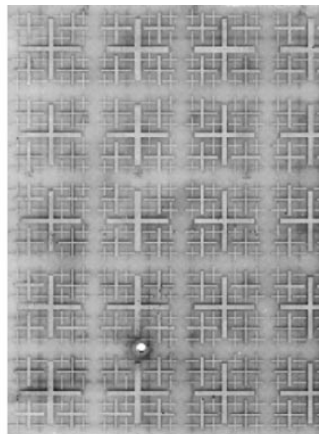

(b)
Fig. 12. (a) Periodic cell of the inset crossed dipole FSS. The grid generates three stopbands that correspond to the three scales of resonant crossed dipoles. The three scales of the dipoles are 3.5, 1.75, and $0.875 \mathrm{~mm}$ thick. (b) Fabricated array on fiberglass.

generation of the cell of an element of the FSS adds a stopband, while maintaining the original response.

The measured far field patterns of the screen in front of a horn antenna are shown in Fig. 11. The contour plots have a field of view of $180^{\circ}$ with the boresight (zero degrees) at the center. The lighter regions represent blockage. It can be seen that the useable angular range for this surface for this size screen is $15^{\circ}$ on either side of the boresight. The cuts of the measured patterns of the horn alone and the horn with the FSS, shown in Fig. 11(b), show how the energy is scattering backward inside the stopbands and allowed to pass through in the passband at $0^{\circ}$. 


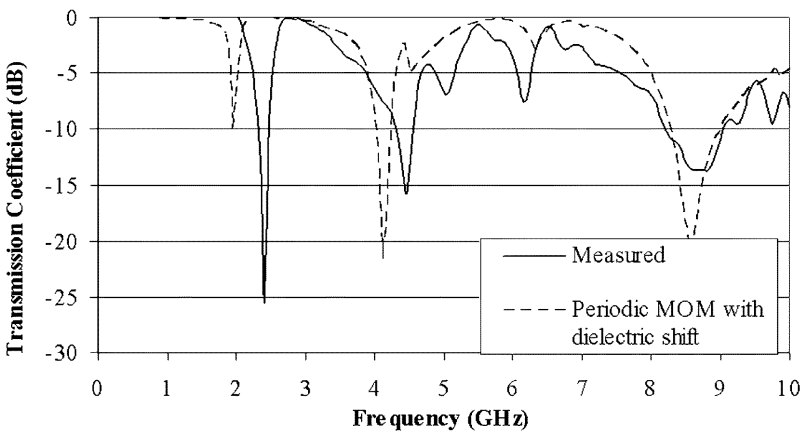

(a)

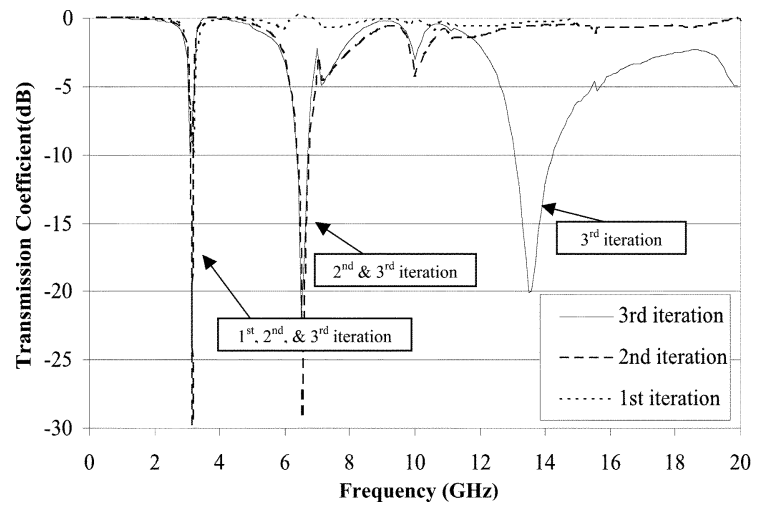

(b)

Fig. 13. (a) Measured frequency response of the inset crossed dipole FSS. The three stopbands corresponding to the three scales in the geometry can be seen. (b) Simulated data of the first three iterations from the periodic MOM technique show how adding iterations to the geometry adds additional resonances.

\section{Inset Crossed Dipole FSS}

1) Inset Crossed Dipole FSS Design: This design is more densely packed than the Sierpinski Carpet fractal FSS. Instead of using resonant patches, resonant crossed dipoles are used. Therefore, the spacing can be tighter. The shift in size between each iteration is one half.

This fractal is generated in a similar manner as the Sierpinski Carpet, where the starting geometry is copied four times and scaled by one half for each iteration. This is expressible as

$$
\begin{aligned}
d_{1,2,3,4}(x, y)= & \frac{1}{2}\left(x \pm \frac{1.5}{6}, y \pm \frac{1.5}{6}\right) \\
D_{S+1}= & D_{S} \cup d_{1}\left(D_{S}\right) \cup d_{2}\left(D_{S}\right) \\
& \cup d_{3}\left(D_{S}\right) \cup d_{4}\left(D_{S}\right)
\end{aligned}
$$

where $D_{s+1}$, the resulting set of points for the $s+1$ iteration is composed of the union of the sets of points resulting from the fours transformations, $d_{1}$ through $d_{4}$. This fractal is limited by the thickness of each dipole. As the number of iterations is increased the spacing between each element in the geometry becomes tighter since this is also scaled with each iteration. Therefore, a thick crossed dipole might not have room to fit between the higher iterations as the number of iterations is increased.

2) Inset Crossed Dipole FSS Performance: Because the scaling of the geometry between each iteration is smaller, two iterations plus the starting geometry can be incorporated and measured in this design. The prefractal element is shown in
Fig. 12(a) and the fabricated array printed on FR4 is shown in Fig. 12(b). Two panels with 5 by 5 elements each have been fabricated and are taped together.

The measured and simulated frequency response can be seen in Fig. 13(a). The three stopbands are present and are spaced with a scaling factor of two between them at approximately 2.2, 4.5, and $9 \mathrm{GHz}$. This superpositioned response shows that multiple resonances can be added to a prefractal FSS by increasing its number of generating iterations. Again the transmission in the passbands is quite lossy and this would need to be corrected depending on the application. The simulated response using the periodic MOM technique distinctly shows the three stopbands. However, the locations of the stopbands are shifted due to inaccuracies in modeling the dielectric shift. The inaccuracies stem from the dielectric being thinner than what is typically considered valid for using an average dielectric shift and furthermore, the dielectric constant of the FR4 dielectric is not well controlled at microwave frequencies.

The periodic MOM simulated responses of the first three iterations of the prefractal as an element of the FSS are shown in Fig. 13(b). These show how the FSS can be designed by superimposing the scaled response onto a previous iteration for the design of multiple resonances. These periodic MOM simulations show the first resonance for the first, second, and third iteration of the fractal to be nearly exact and likewise for the second resonance for the second and third iteration of the fractal.

The measured far field patterns are shown in Fig. 14 for the five bands of interest, which are defined to be the three stopbands and the two transmission bands that fall between the stopbands. As with the Sierpinski Carpet fractal FSS, it can be seen that the useable angular range for this surface for this size screen is $15^{\circ}$ on either side of the boresight. The contour plots have a field of view of $180^{\circ}$ with the boresight (zero degrees) at the center. The lighter regions represent blockage and therefore show the angles for which the FSS is effective. Two representative cuts are shown in Fig. 14(b) which show the energy being passed at $0^{\circ}$ for the cut inside the passband on the right and the energy being scattered backward for the cut inside stopband shown on the left when the patterns with and without the FSS are compared.

\section{CONCLUSION}

Three new cells based on the concept of fractals that can be used to build a periodic FSS have been proposed. They are dual polarized and have a multiband behavior which has been numerically and experimentally verified. It has been shown how fractals can play a role in electromagnetic designs even at low levels of generating iterations due to the inherent self-similarity in the geometry. The designs are simulated using a periodic MOM with an average dielectric shift as well as a periodic FDTD method. The prefractal surfaces have been fabricated and measured to verify the frequency and angular response. The correlations between the geometrical scaling and the spacing between the frequency bands of its response are compared in Table I. The Sierpinski carpet and the inset crossed dipoles led to good correlation between the geometry and the expected stopbands. The inset crossed dipoles 


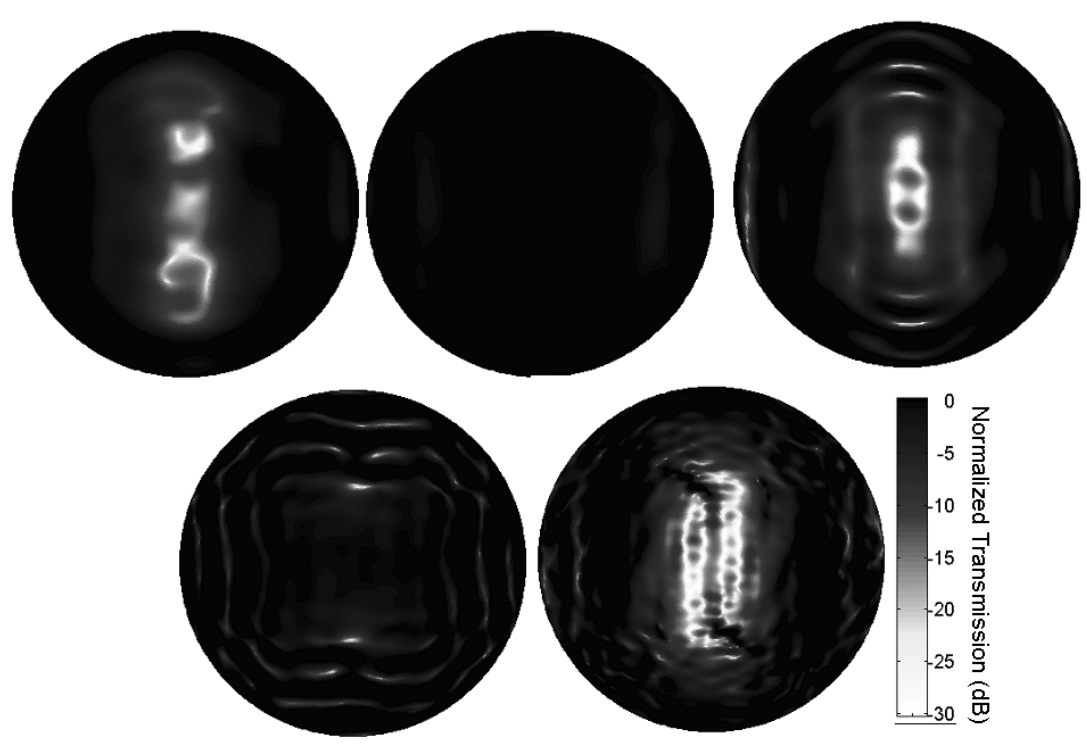

(a)
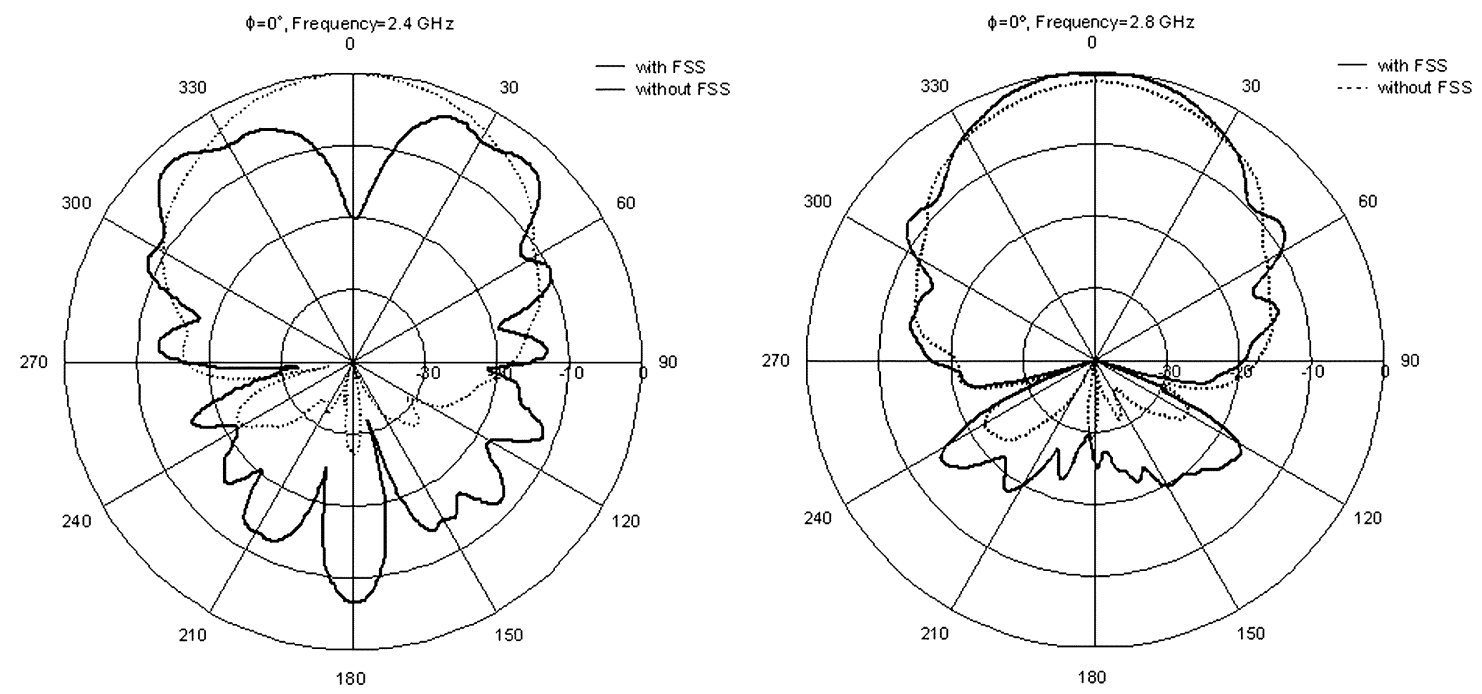

(b)

Fig. 14. Measured far field patterns for the inset crossed-dipole FSS. The five patterns in (a) represent the five frequency bands of interest starting from the top left: first stopband, first passband, and second stopband and on the bottom from the left: second passband and the third stopband. (b) Cuts of the first stopband and first passband.

TABLE I

COMPARISON BETWEEN THE SCALING IN THE GEOMETRIES AND THE SPACING BETWEen THE RESUlting FREQUENCY BANDS FOR EACH PRE-FraCtAl FSS

\begin{tabular}{l|c|c|c|c}
\hline & $\begin{array}{c}\text { Geometrical } \\
\text { Spacing }\end{array}$ & $\begin{array}{c}\text { Spacing Between } \\
\text { Frequency Bands }\end{array}$ & $\begin{array}{c}\text { Measured } \\
\text { Transmission } \\
\text { Coefficient in } \\
\text { Pass Bands }\end{array}$ & $\begin{array}{c}\text { Measured } \\
\text { Transmission } \\
\text { Coefficient in } \\
\text { Stop Bands }\end{array}$ \\
\hline Minkowski & 2.5 & 3.67 & $0 \mathrm{~dB}$ & $-27 \mathrm{~dB}$ \\
Fractal & & & & $-1 \mathrm{~dB}$ \\
\hline Sierpinski Carpet & 4 & 4 & & $-30 \mathrm{~dB},-19 \mathrm{~dB}$ \\
Fractal & & & $0 \mathrm{~dB},-1 \mathrm{~dB}$ & $-25 \mathrm{~dB},-16 \mathrm{~dB}$, \\
\hline Inset Crossed- & 2 & 2 & & $-14 \mathrm{~dB}$ \\
\hline
\end{tabular}

allowed the tightest spacing between resonances. Tradeoffs between the periodicity, starting geometry, and supporting dielectrics can improve performance in the passbands and allow for bands of various number and spacing.

\section{ACKNOWLEDGMENT}

The authors would like to acknowledge the measurement and fabrication assistance provided by the engineering staff at the Universitat Politécnica de Catalunya.

\section{REFERENCES}

[1] H. Peitgen, H. Jürgens, and D. Saupe, Chaos and Fractals: New Frontiers of Science. New York: Springer-Verlag, 1992.

[2] C. Puente, J. Romeu, R. Pous, X. Garcia, and F. Benitez, "Fractal multiband antenna based on the Sierpinski gasket," Electron. Lett., vol. 32, no. 1, pp. 1-2, Jan. 4, 1996.

[3] J. Romeu and Y. Rahmat-Samii, "Dual band FSS with fractal elements," Electron. Lett., vol. 35, no. 9, pp. 702-703, Apr. 29, 1999. 
[4] - "Fractal FSS: a novel dual-band frequency selective surface," IEEE Trans. Antennas Propagat., vol. 48, pp. 1097-1105, July 2000.

[5] V. K. Varadan, K. J. Vinoy, K. A. Jose, and V. V. Varadan, "Conformal fractal antennas and FSS for low RCS applications," in Proc. SPIE-Int. Soc. Optical Engineering, vol. 3990, March 2000, pp. 138-145.

[6] D. H. Werner and D. Lee, "Design of dual-polarized multiband frequency selective surfaces using fractal elements," Electron. Lett., vol. 36, no. 6, pp. 487-488, March 16, 2000.

[7] J. P. Gianvittorio, Y. Rahmat-Samii, and J. Romeu, "Fractal FSS: various self-similar geometries used for dual-band and dual-polarized FSS," in Proc. IEEE Antennas and Propagation Soc. Int. Symp., vol. 3, 2001, pp. 640-643.

[8] P. P. Sarkar, D. Sarkar, S. Das, S. Sarkar, and S. K. Chowdhury, "Experimental investigation of the frequency-selective property of an array of dualtuned printed dipoles," Microw. Opt. Tech. Lett., vol. 31, no. 3, pp. 189-190, Nov. 5, 2001.

[9] A. S. Barlevy and Y. Rahmat-Samii, "Characterization of electromagnetic bandgaps composed of multiple periodic tripods with interconnecting vias: concept, analysis, and design," IEEE Trans. Antennas Propagat., vol. 49, Mar. 2001.

[10] Y. Rahmat-Samii and H. Mosallaei, "Electromagnetic bandgap structures: classification, characterization, and applications," in Proc. ICAP-11th Int. Conf. Antennas and Propagation, vol. 2, Apr. 2001, pp. $560-564$.

[11] A.Akira Ishimaru, Electromagnetic Wave Propagation, Radiation, and Scattering. Englewood Cliffs: Prentice Hall, 1991

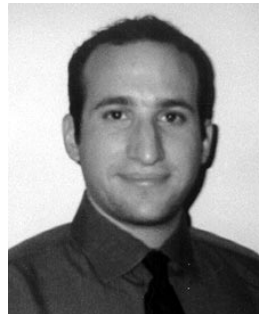

John P. Gianvittorio was born in Philadelphia, PA, in January 1976. He received the BSEE degree from Drexel University, Philadelphia, in 1998, after completing a five year co-operative education program that included three six month co-ops in various telecommunication and defense electronic companies. He received the MSEE degree from the University of California, Los Angeles with a thesis entitled "Fractal Antennas: Design, Characterization and Applications," where he is currently working toward the Ph.D. degree.

He has worked in the Antenna Research, Analysis, and Measurement Laboratory at UCLA under the direction of Professor Yahya Rahmat-Samii since September of 1998. He has worked on various projects involving fractal antennas including fabrication and measurements of antenna elements and phased arrays and has presented his research at various antenna conferences. In 2002, he presented his research and antenna fundamentals as a Guest Lecturer at the National University of Defense Technology in Changsha, P.R. China. He engaged in research for three months starting in April of 2001 at the Universitat Politecnica de Catalunya in Barcelona, Spain with the Department of Signal Theory and Communications under the direction of Professor Jordi Romeu in the area of fractal frequency selective surfaces. He has also worked in conjunction with the MEMS research group at UCLA in a project combining electromagnetics and MEMS technologies.

Mr. Gianvittorio is a Member of Alpha Pi Lambda, at Drexel University, and Tau Beta Pi and Eta Kappa Nu, engineering and electrical engineering honor societies. He was awarded the Outstanding MS Student Award for 1999-2000 from the Electrical Engineering Department, UCLA, a Travel Grant for Young Investigators from Spike Broadband Systems, Incorporated, in 2002, and the Best Presentation Award in the 2002 UCLA Electrical Engineering Annual Research Review.

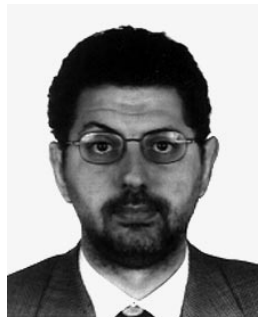

Jordi Romeu (S'88-M'93) was born in Barcelona, Spain, in 1962. He received the Ingeniero and Doctor Ingeniero degrees in Telecommunication Engineering from the Polytechnic University of Catalonia (UPC), Barcelona, Spain, in 1986 and 1991, respectively.

In 1985, he joined the Electromagetic and Photonics Engineering Group of the Signal Theory and Communications Department, at UPC. Currently, he is an Associate Professor at UPC, where he is engaged in research in antenna near field measurements, antenna diagnostics and antenna design. He was a Visiting Scholar at the Antenna Lab at the University of California, Los Angeles, in 1999 with a NATO Scientific Program scholarship. He holds several patents and has published papers in the fields of antenna near-field measurements and diagnostics, and in antenna design.

Dr. Romeu was the Grand Winner of the 1998 European IT prize awarded by the European Commission for his contributions in the development of fractal antennas.
Sebastián Blanch was born in Barcelona, Spain, in 1961. He received the Ingeniero and Doctor Ingeniero degrees in telecommunication engineering from thePolytechnic University of Catalonia (UPC), Barcelona, Spain, in 1989 and 1996 , respectively.

In 1989, he joined the Electromagnetic and Photonics Engineering Group of the Signal Theory and Communications Department. Currently, he is an Associate Professor at UPC. His research interests are antenna near field measurements, antenna diagnostics, and antenna design.

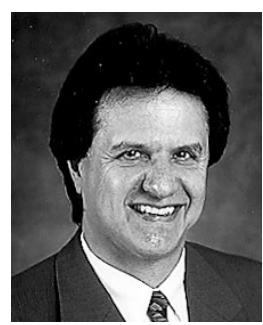

Yahya Rahmat-Samii (S'73-M'75-SM'79-F'85) received the M.S. and Ph.D. degrees in electrical engineering from the University of Illinois, Urbana-Champaign.

$\mathrm{He}$ is a Professor and the Chairman of the Electrical Engineering Department at the University of California, Los Angeles (UCLA). He was a Senior Research Scientist at NASA's Jet Propulsion Laboratory/California Institute of Technology before joining UCLA in 1989. He was a Guest Professor at the Technical University of Denmark (TUD) in the summer of 1986. He has also been a consultant to many aerospace companies.

Dr. Rahmat-Samii was elected Vice-President and President of the IEEE Antennas and Propagation Society in 1994 and 1995, respectively. He was appointed an IEEE Antennas and Propagation Society Distinguished Lecturer and presented lectures internationally. He was elected as a Fellow of the Institute of Advances in Engineering (IAE) in 1986. He was also a member of the Strategic Planning and Review Committee (SPARC) of IEEE. He was the IEEE AP-S Los Angeles Chapter Chairman (1987-1989); his chapter won the best chapter awards in two consecutive years. He has been the plenary and millennium session speaker at many national and international symposia. He has been the organizer and presenter of many successful short courses worldwide. He was one of the Directors and Vice President of the Antennas Measurement Techniques Association (AMTA) for three years. He has been Editor and Guest Editor of many technical journals and book publication entities. He has also served as Chairman and Co-Chairman of several national and international symposia. He was also a member of UCLA's Graduate Council for a period of three years. For his contributions, he has received numerous awards. He has received many NASA and JPL Certificates of Recognition. In 1984, he received the coveted Henry Booker Award of the International Scientific Radio Union (URSI) from the International Union of Radio Science, which is given triennially to the most outstanding young radio scientist in North America. Since 1987, he has been designated every three years as one of the Academy of Science's Research Council Representatives to the URSI General Assemblies held in various parts of the world. He was also the invited speaker to address the URSI 75th Anniversary in Belgium. In 1992 and 1995, he was the recipient of the Best Application Paper Prize Award (Wheeler Award) for papers published in the 1991 and 1993 IEEE TRANSACTIONS ON ANTENNAS AND PROPOGATION. In 1993 to 1995, three of his Ph.D. students were named the Most Outstanding Ph.D. students at UCLA's School of Engineering and Applied Science. Six others received various Student Paper Awards at the 1993 to 2001 IEEE AP-S/URSI Symposiums. He is a Member of Commissions A, B, J, and K of USNC/URSI, AMTA, Sigma Xi, Eta Kappa Nu, and the Electromagnetics Academy. He is listed in Who's Who in America, Who's Who in Frontiers of Science and Technology, and Who's Who in Engineering. In 1999, he was the recipient of the University of Illinois ECE Distinguished Alumni Award. In 2000, he was the recipient of IEEE Third Millennium Medal and AMTA Distinguished Achievement Award. In 2001, he received the prestigious Honorary Doctorate from the University of Santiago de Compostela, Spain. In 2001, he was elected as a Foreign Member of the Royal Flemish Academy of Belgium for Science and the Arts. He is the designer of the IEEE Antennas and Propagation Society logo that is displayed on all IEEE ANTENNAS AND PROPAGATION publications. 